\title{
ANALYSIS OF SCHEMES OF CONTROL AND MANAGEMENT OF GLASS ROD DRAWING PROCESS
}

\author{
L B Alekseeva \\ Saint-Petersburg Mining University \\ 21 line, 2, St. Petersburg, 199106, Russia \\ lbalek@rambler.ru
}

\begin{abstract}
The transient processes, associated with the management process of drawing glass rods, are observed. To solve this problem, mathematical models are used; the zones, in which the glass rod (light guide) is formed, the actuator, including a motor, a transmission mechanism. These models allow us to determine the nature of transient processes, to select the control method and to determine the requirements for the drawing mechanism. The conditions of the process flow stability are found. This determines the sensitivity of the control object to small perturbations. The methods of perturbation and deviation are observed. For example, when the work process runs without overshoot, an excessive rate method is applicable. In case of overshoot, it is better to use quasi-optimal control. Conditions of most common aperiodic and oscillation regimes are determined.
\end{abstract}

Keywords-glass rod, drawing, mechanism, model, management, transition mode, perturbation.

\section{INTRODUCTION}

The object of research is the process of drawing glass rods. The main objective of the work - analysis of methods and means of process control that enhance the quality of products.

To solve management problems, the static and dynamic properties of the management object must be considered. Static properties are determined by the dependence of the output quantity on the input value in the steady state. These properties determine the sensitivity of the control object to small perturbations and predetermine the range for deviation tolerances in basic technological parameters depending on the requirements for output parameters [1, 2]. Dynamic characteristics determine the dependence of the output quantity on the input values in the transient regimes that arise during process control.

\section{MANAGEMENT OF PERTURBATION OR DEVIATION}

For the technological process of drawing light guides, one can use control systems shown in fig. 1 for perturbation and deviation.

In the process under study, perturbations of any kind lead to a change in the configuration of the formation zone and the pulling force.

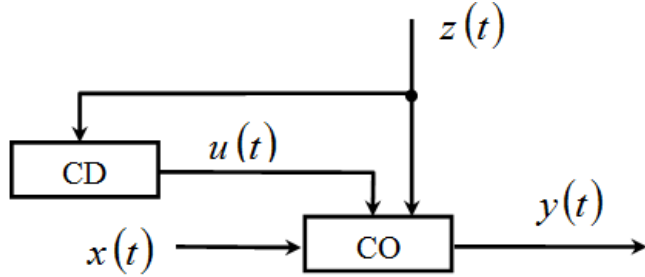

b)

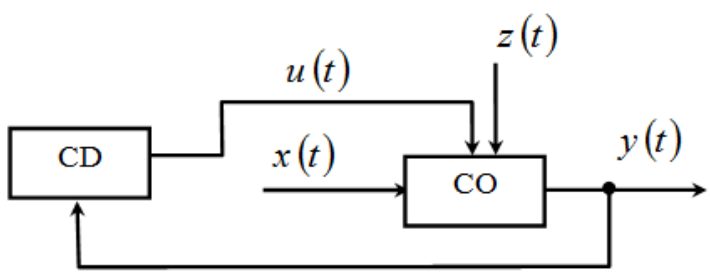

Fig. 1. Management: $a$-of the perturbation; $b-$ of the deviation

In the image, there are the following labels: $\mathrm{CO}$ - control object; CD - control device, $x(t)$ - control command (input, such as the drawing speed); $y(t)$ - controlled variable (yield, the diameter of the glass rod); $u(t)$ - control action.

In management, parameter $z(t)$ is controlled, (e.g., the pulling force), which instantly reacts to changes occurring within the control object itself. This is the main advantage of such a management structure. However, as the output parameter of the glass rod is not controlled, it requires the identification of the relationship between changes in pulling force and a glass rod diameter.

\section{PROCESS CONTROL}

The complexity of implementing such a scheme is the continuity of the pulling process, and for rigid glass rods, it is impossible to bend them at large angles in order to measure the pulling force.

Depending on the sensor used for measuring the pulling forces, devices can be divided into mechanical, optical, electrical. In the mechanical devices, pulling forces are recorded using mechanical type sensors (counting by means of limbs, indicators). The advantage of mechanical devices is that 
they do not require complex equipment for converting mechanical displacements into electrical signals. In optical devices, the displacements, associated with changes in pulling force, are converted into an optical signal. In electrical devices, transducers convert mechanical stresses into an electrical signal.

The pull force for rigid glass rods may be $10 \ldots 50 \mathrm{~N}$ with an accuracy of $\pm 0.05 \%$. Circuits using a direct piezoelectric effect can provide such accuracy.

When controlling perturbations, output parameter $y(t)$ is constantly monitored. Management is formed based on the results of such control. In this case, the so-called transport lag problem arises. Information about the state of the control object cannot be obtained in real time. Consequently, it is necessary to find the dependencies that allow determining the state of the management object at certain subsequent instants of time, if the management and perturbation effects in the preceding moments are known. These dependencies allow one to search for the optimal control parameter for particular values. In this case, the control signal is continuously developing and must match the optimum parameter (adaptive management).

\section{THE MATHEMATICAL MODEL OF THE DRIVE}

When controlling, transients occur, which enable one to determine the management method. To do this, one should select a model on which it is possible to base the decisionmaking procedure.

This type of the drive includes a motor and a gearbox. The links of the drive mechanisms are absolutely rigid; the author does not take into account gaps in kinetic pairs. The mechanism has one degree of freedom. All forces and weights go to the output link.

Let us examine the character of the forces acting on the links. The motor's dynamic characteristics are [3]:

$$
\tau_{\mathrm{m}} \frac{d M_{\mathrm{m}}}{d t}+M_{\mathrm{m}}=M_{\mathrm{m} S},
$$

where $\tau_{\mathrm{m}}$ - electromagnetic time constant of the motor; $M_{\mathrm{m} S}, M_{\mathrm{m}}-$ given moments of forces of resistance and driving forces.

Value $\tau_{\mathrm{m}}$ determines the inertia of electromagnetic processes occurring in the engine and in its control system.

The author uses a DC motor. For the type of the engine under consideration, the static characteristic $M_{\mathrm{m} S}$ is linear

$$
M_{\mathrm{m} S}=M_{0}-k \omega_{\mathrm{m}},
$$

where $M_{0}$-engine starting torque; $k$-steepness of the static characteristic of the engine.

It is necessary to send all the force and weight of mobile parts of the actuator mechanism to its output link (star point transmission chain, belt pulley, friction roller, etc.). Then the equation of motion of the drawing mechanism in differential form will be [4]:

$$
I(\varphi) \frac{d \omega}{d t}+\frac{1}{2} \frac{d I}{d \varphi} \omega^{2}=M_{\mathrm{m}}-M_{\mathrm{c}},
$$

where $I-$ the given moment of inertia; $\omega=\frac{d \varphi}{d t}-$ the angular velocity of the output link of the drive mechanism (actuating unit).

Thus, the dynamic model corresponds to the mathematical model, and consists of two equations (1) and (2) which can be reduced to one equation (for $I=$ const) [5]:

$$
\tau_{\mathrm{d}} \tau_{\mathrm{m}} \frac{d^{2} \omega}{d t^{2}}+\tau_{\mathrm{d}} \frac{d \omega}{d t}+\omega=\frac{1}{k}\left(M_{0}-M_{\mathrm{c}}-\tau_{\mathrm{m}} \frac{d M_{\mathrm{c}}}{d t}\right),
$$

where $\tau_{\mathrm{d}}=I / k$ is the constant time of the drive

The right side of equation (3) defines the speed value $\omega_{2}$ of the link, on which the drive is reconfigured after the control signal is applied to the motor.

\section{THE PARAMETERS DEFINING THE NATURE OF THE TRANSITIONAL DRIVE MODES}

Let us introduce the dimensionless parameters:

$$
\psi=\frac{t}{\sqrt{\tau_{\mathrm{d}} \tau_{\mathrm{m}}}}, \quad \varsigma=\frac{1}{2} \sqrt{\frac{\tau_{\mathrm{d}}}{\tau_{\mathrm{m}}}},
$$

and reduce equation (3) to the form:

$$
\frac{d^{2} \omega}{d \psi^{2}}+2 \varsigma \frac{d \omega}{d \psi}+\omega=\omega_{2} .
$$

The solution of equation (4) and, accordingly, the nature of the transient processes depend on the ratio of $\tau_{\mathrm{d}}$ and $\tau_{\mathrm{m}}$, that is, on $\zeta$.

When $\varsigma>1$, the general solution of equation (4) has the form:

$$
\omega=\omega_{2}+A_{1} \exp \left(\lambda_{1} \psi\right)+A_{2} \exp \left(\lambda_{2} \psi\right),
$$

where $A_{1}, A_{2}-$ constants that depend on the initial conditions; $\lambda_{1}, \lambda_{2}$ are the roots of the characteristic equation.

Supposing that $\psi=0$

$$
\omega=\omega_{1} ; M_{\mathrm{m}}=M_{0}^{*}-k\left(\omega_{1}-\omega_{2}\right),
$$

where $M_{0}^{*}$ is the starting torque corresponding to speed $\omega_{2}$.

Using (6), it is possible to obtain:

$$
\omega_{1}-\omega_{2}=A_{1}+A_{2} \text {. }
$$

After the transformations, $A_{1}$ and $A_{2}$ are found:

$$
A_{1}=\frac{\Delta}{2 \varsigma} \cdot \frac{2 \varsigma \lambda_{2}+1}{\lambda_{1}-\lambda_{2}}, A_{2}=\frac{\Delta}{2 \varsigma} \cdot \frac{2 \varsigma \lambda_{1}+1}{\lambda_{2}-\lambda_{1}},
$$

where $\Delta=\omega_{2}-\omega_{1}$ - speed adjustment range $\omega$.

For the convenience of representation of (5) in a dimensionless form, let us subtract $\omega_{1}$ from the left and right parts of this expression. After the transformation, one obtains:

$$
\frac{\Delta_{\omega}}{\Delta}=1+\bar{A}_{1} \exp \left(\lambda_{1} \psi\right)+\bar{A}_{2} \exp \left(\lambda_{2} \psi\right),
$$

where $\overline{A_{1}}=A_{1} / \Delta ; \bar{A}_{2}=A_{2} / \Delta ; \Delta_{\omega}=\omega-\omega_{1}$.

Let us consider the nature of the transient process at $\varsigma<1$. In this case, the roots of the characteristic equation are complex conjugate and the general solution of equation (4) should be searched for in the form of (5). 
Using the initial conditions, after similar transformations, one obtains:

$$
\frac{\Delta_{\omega}}{\Delta}=1+\bar{A}_{3} \exp (-\varsigma \psi) \cos \left(\sqrt{1-\varsigma^{2}} \psi+\alpha\right),
$$

where $\bar{A}_{3}=-\frac{1}{2 \varsigma \sqrt{1-\varsigma^{2}}}$.

The analysis of expressions (9), (10) shows that in this case, the transient process passes with overshooting and has the character of damped oscillations.

The over-regulation of the process can be avoided by introducing a quasi-optimal management [6]. For this, the voltage applied to the motor's armature changes in jumps.

\section{PROCESESS IN THE FORMATION ZONE}

Transient processes in the drive create a perturbing force acting on the ongoing technological process (drawing of products, shaping during machining, etc.). (A zone of product formation). Let us assume that the nature of change in this force corresponds to the character of the change in engine speed:

$$
F(t)=\Delta F \Omega,
$$

where $\Delta F=F_{2}-F_{1} ; F_{1}, F_{2}-$ the magnitude of the forces acting on the technological process, corresponding to $\omega_{1}, \omega_{2}$ speeds, $\Omega=\Delta_{\omega} / \Delta$ and determined by the expressions (9), (10), (11).

Going forward, as an example, let us consider the technological process of drawing products from softened glass mass [7], [8].

The transient process, associated with the impact on the force forming zone $F(t)$, will be determined from the change of length $L$ of formation zones. $L-L_{0}=z(t), L_{0}-$ original length of the formation zone.

The transitional process can be defined based on the Duhamel-Carson integral:

$$
z(t)=\int_{0}^{t} F(\tau) h(t-\tau) d \tau
$$

where $\tau$ - supporting integrated time, varying from zero to considered current time $t ; h(t-\tau)$ - glass reaction at time $t$ for a single pulse.

To calculate reaction $h(t-\tau)$, a linear equation of the form is used:

$$
m \frac{d^{2} z}{d t^{2}}+\eta \frac{d z}{d t}+c z=0
$$

where $m$ - amount of mass in the formation zone; $\eta, c-$ coefficients corresponding to damping and elasticity.

Let us take into account only the viscous properties of the glass mass and determine the response to single action $f(t)$. Apriory:

$$
f(t)=\left\{\begin{array}{l}
1(t) \text { when } t \geq 0 ; \\
0 \text { when } t<0 .
\end{array}\right.
$$

Equation (13) takes the form:

$$
m \frac{d^{2} z}{d t^{2}}+\eta \frac{d z}{d t}=1(t)
$$

Let us form the characteristic equation for (14):

$$
m \gamma^{2}+\eta \gamma+c=0
$$

The roots of this equation are equal to:

$$
\gamma_{1,2}=-\frac{\eta}{2 m} \pm \sqrt{\left(\frac{\eta}{2 m}\right)^{2}-\frac{c}{m}} \text {. }
$$

To estimate the roots from (15), and, therefore, to describe the transient process, it is convenient to introduce the concept of critical factor damping [9] equal to:

$$
\eta_{c r}=2 m \sqrt{\frac{c}{m}}=\frac{4 m \pi}{T} .
$$

where $T=2 \pi \sqrt{m / c}$.

Let us introduce relation $\eta / \eta_{c r}=n$. Then (15) can be represented in the form:

$$
\gamma_{1,2}=\frac{2 \pi}{T}\left(-n \pm \sqrt{n^{2}-1}\right) .
$$

When $n>1$, the behavior of the glass mass corresponding to the aperiodic link of the glass mass in the formation zone has a viscous nature.

When $n<1$, the behavior of the glass mass corresponds to the typical oscillatory links.

\section{MODES THAT OCCUR DURING CONTROL IN THE DRIVE SYSTEM - TECHNOLOGICAL PROCESS}

This character depends on the parameters and their relationships. The most typical variants were examined, for example, if $n>1$ and $\varsigma>1$, there is an aperiodic mode.

The proposed approach allows one to take into account the parameters of the drive and the technological process (formation zone) and choose the control method by changing the motor speed. For example, if the process occurs without overshooting, one can use the forced transition method. If the process takes place with overshooting, then it is possible to use quasi-optimal control "fig. 2".

a)

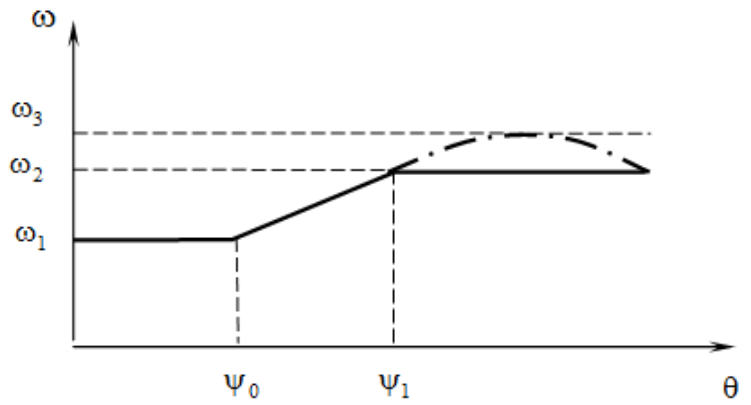


b)

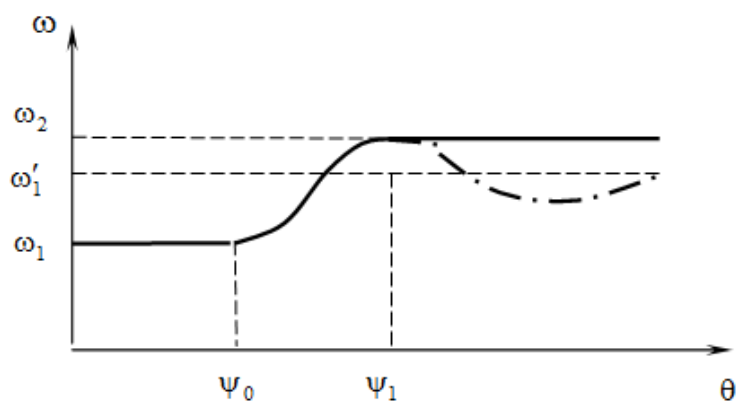

Fig. 2. Methods of regulation of the engine speed: $a$ - forced; $b$ - quasi-optimal

To assess the stability of an automated control system (ACS), it is necessary to determine the transfer function. For the considered technological process, that function has the form [10]:

$$
P=\frac{W_{\mathrm{CD}} W_{\mathrm{CO}}}{1+W_{\mathrm{CD}} W_{\mathrm{CO}}},
$$

where $W_{\mathrm{CD}} \quad W_{\mathrm{CO}}-$ the transfer functions controlling the device and the control object, respectively.

The main elements of the control device that determine the nature of the transient processes are the electric drive and the extraction mechanism. The glass rod drawing process is usually carried out by adjusting the speed of the output link in the drawing mechanism.

Usually, as the author already noted, DC electric motors with a linear mechanical characteristic are used in the electric drives of the drawing devices.

The transfer functions are of the form:

$$
\begin{array}{r}
W_{\mathrm{CD}}=\left(T_{1} p^{2}+T_{2} p+1\right)^{-1} ; \\
W_{\mathrm{CO}}=\left(T_{3} p^{2}+T_{4} p+1\right)^{-1},
\end{array}
$$

where $T_{1}=\tau_{\mathrm{d}} \tau_{\mathrm{m}} ; T_{2}=\tau_{\mathrm{d}} ; T_{3}=m / c ; T_{4}=\eta / c$; an operator is in transformation of Laplace, equal $p^{n}=d^{n} / d t^{n}$.

After the transformations, one obtains the characteristic equation of the fourth order:

$$
a_{4} p^{4}+a_{3} p^{3}+a_{2} p^{2}+a_{1} p+a_{0}=0,
$$

where $\quad a_{4}=T_{1} T_{3}, \quad a_{3}=T_{1} T_{4}+T_{2} T_{3}, \quad a_{2}=T_{1}+T_{2} T_{4}+T_{3}$, $a_{1}=T_{2}+T_{4}, a_{0}=2$.

To analyze the stability of the system, let us use the algebraic Routh-Hurwitz criterion. The system under consideration is stable if following conditions are met:

1. All the coefficients of the equation (20) must be positive.

2. $\Delta_{2}=\left|\begin{array}{ll}a_{2} & a_{0} \\ a_{3} & a_{1}\end{array}\right|=a_{2} a_{1}-a_{0} a_{3}>0$.

3. $\Delta_{3}=\left|\begin{array}{lll}a_{3} & a_{1} & 0 \\ a_{4} & a_{2} & a_{0} \\ 0 & a_{3} & a_{1}\end{array}\right|=a_{1}\left(a_{3} a_{2}-a_{1} a_{4}\right)-a_{3}^{2} a_{0}>0$.
The first condition for the system under consideration is unconditionally satisfied. The second condition in the assumed dimensionless parameters takes the form:

$$
1+8 \frac{n \varsigma^{2}}{\tau}+16 \frac{n^{2} \varsigma^{2}}{\tau^{2}}+2 \frac{n \varsigma^{2}}{\pi^{2} \tau^{2}}>+\frac{2 n}{\tau} \frac{\varsigma^{2}}{\pi^{2} \tau^{2}},
$$

where $\tau=\tau_{\mathrm{d}} / T$.

It is possible to highlight areas in parameters $n, \varsigma, \tau$, in which this condition is certainly satisfied:

$$
n>\tau / 2, \quad \varsigma^{2}>0,25 /\left(1+\frac{n}{\tau / 2}\right) .
$$

The third condition in the dimensionless parameters has the form:

$$
\left(\frac{\pi^{2} \tau^{2}}{\varsigma^{2}}+4 \pi \tau n+4 n^{2}\right)+\varsigma^{2}\left(4+\frac{4 n}{\pi \tau}+\frac{1}{\pi^{2} \tau^{2}}\right)>\frac{\varsigma^{2}}{\pi \tau n}+\frac{\pi \tau n}{\varsigma^{2}}
$$

So, if $n^{2}>0,25$ and $\varsigma^{2}>0,25$, then the system is stable for all values of $\tau$.

In order to identify the impact of $\tau$, let us propose inequality (23) in the form:

$$
y(z)=A z^{2}+B z+C>0,
$$

where $z=\varsigma^{2} ; \quad A=4+\frac{4 n}{\pi \tau}+\frac{1}{\pi^{2} \tau^{2}}-\frac{1}{\pi \tau n} ; \quad B=4 \pi \tau n+4 n^{2}$; $C=\pi^{2} \tau^{2}-\pi \tau n$

Because $A>0$, the branches of parabola $y(z)$ are directed upwards. In this case, if $C<0$ ( or $n>\pi \tau$ ), then equation $y(z)$ $=0$ has one positive root $z_{1}$. Then the system is stable under $z>z_{1}$. Thus, inequalities $n>\pi \tau$ and $z>z_{1}$ specify the area of parameters $n, \varsigma, \tau$, in which the system is stable.

\section{EVALUATION OF THE QUALITY OF CONTROL}

Stability is related to the behavior of the system at infinity, and real systems operate on a finite time interval. In this case, let us use the concept of the quality of the system, proposed by V. V. Solodovnikov. The system can be regarded as satisfying the required quality if the transfer function does not go beyond certain areas ("boxes"). One of the direct indicators of the quality of the system is the time of the transient process. The expressions obtained above, determine the nature of the transition processes, allow building algorithms for all the above cases. When there is a need to change the regime, the motor is supplied with a voltage corresponding to speed $\omega_{3}$, that is, more than what is necessary to achieve speed $\omega_{2}$. At the same time, $\omega$ is forced to increase. With $\psi_{1}$, when $\omega$ reaches the required value of $\omega_{2}$, the voltage must be reduced stepwise up to the value corresponding to speed $\omega_{2}$. Difference $\psi_{1}-\psi_{0}=\psi_{\mathrm{tp}}$ defines the time of the transient process.

Let us examine the most common case when $n>1, \varsigma>1$. The graphs in fig. 3, show the influence of parameters $n$, $\tau_{\mathrm{d}} / T$ on the time of the transient process. With growth of $n$, the size of $\psi_{\text {tp }}$ increases, but with growth of $\tau_{\mathrm{d}} / T$, it 
decreases. The graph in fig. 4, shows the effect of the parameter $\tau$ during the transition process. The analysis showed that $\psi_{\text {tp }}$ has a final limit at $\tau_{\mathrm{d}} / T \rightarrow \infty$.

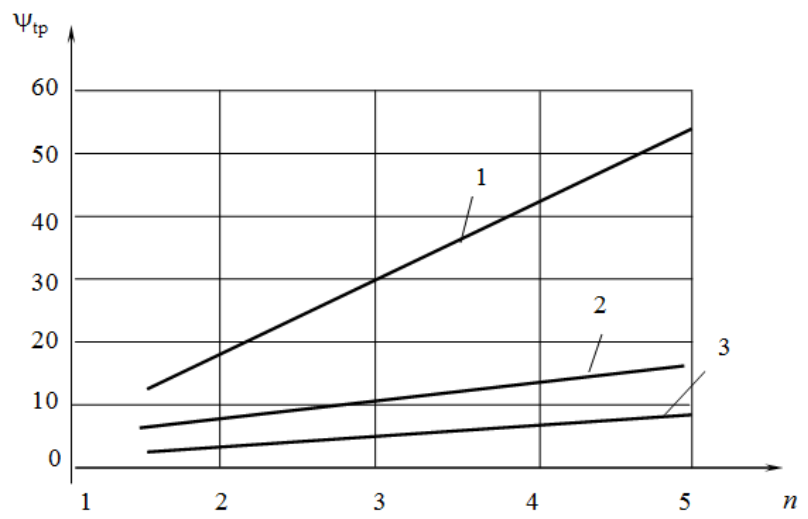

Fig.3.The impact on the time of transient process parameters $n, \tau_{\mathrm{d}} / T$ at $\varsigma=1,5$. $1-\tau_{\mathrm{d}} / T=0,2 ; 2-\tau_{\mathrm{d}} / T=0,7 ; 3-\tau_{\mathrm{d}} / T=1,5$

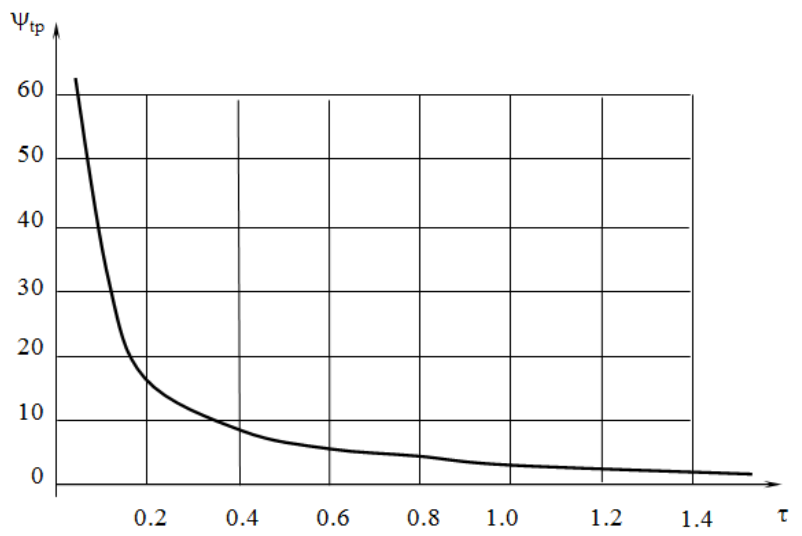

Fig.4. The impact on the time of transient process parameter $\tau$ at $n=\varsigma=1,5$.

\section{DRAWING MECHANISMS}

The most important direction is the improvement of the main drawing assembly - a drawing mechanism. In most management schemes, this mechanism is the regulator. Conflicting requirements are often imposed on the drawing mechanism. It should provide a stable environment for the drawing process, and, at the same time, react instantly to a deviation of the controlled parameter.

The research enabled the formulation of basic guidelines for the development of drawing devices, for example, management of ineffective deviation due to large lags and inertia of the process. The perturbation control requires the development of drawing systems with control systems for the extraction force. In addition, the extraction mechanism should create the least interference caused by vibrations of moving parts.

To implement the control circuitry, direct and indirect methods of measuring the geometrical characteristics (the diameter, the thickness of the walls of the capillary) of the light guide are used.

The device in fig. 5 comprises inner 1 and outer 2 containers, installed in furnace 3. Glass mass level sensors are mounted in receptacles 4,5 , which via control units 6,7 are electrically connected to electric motors 8 and 9 which regulate pneumatic pumps 10,11 . Under the vessels, there is sensor 12 with cross-sectional dimensions of glass rod 13, drawn out from the vessels by drawing mechanism 14. Sensor 12 is electrically connected with the electric motors of pneumatic pumps through control blocks 6,7 .

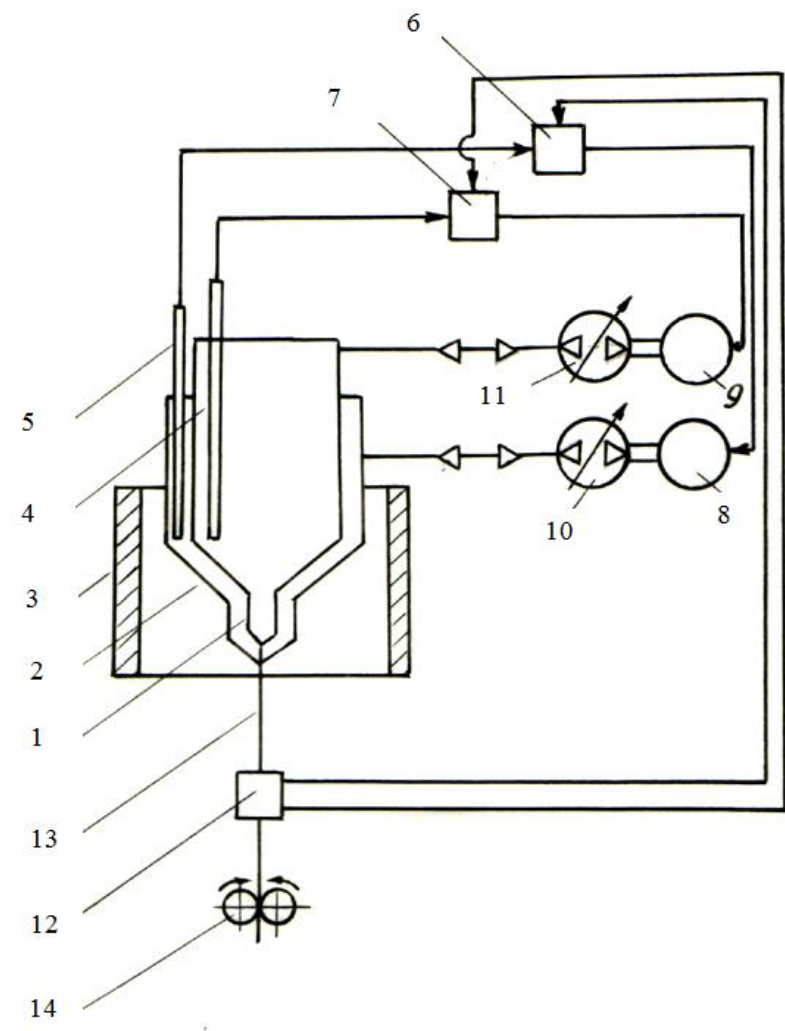

Fig. 5. Drawing apparatus with control of glass rod geometry

The principle of the device function. Core glass is placed in the vessel 1 , in the outer vessel 2 -glass for the fiber shell. Then the glass is heated by furnace 3 to the softening point and glass rod 13 is drawn with drawing mechanism 14. As the glass level in the vessels drops, a signal from sensors 4,5 comes via control units 6,7 to electric motors 8,9 , and then to pneumatic pumps 10, 11 which feed gas into the vessels, increasing the pressure therein according to a predetermined law, so that the hydrostatic head on the bottom of the vessel remains at a given level. Sensor 12 monitors the core and cladding diameters of the glass rods. For this purpose, such proximity sensors as lasers are used.

\section{CONCLUSION}

Mathematical models of the control unit and the control object for the study of transient regimes that arise in process control are proposed.

The influence of the system parameters on the nature of the transient regimes is evaluated, which allows choosing the control method and determining the time of the transient regimes. 
The influence of parameters on the stability of the motion of the system is determined.

On the basis of theoretical studies, directions for the future development of drawing devices have been determined, in accordance with which a number of schematic technical solutions have been proposed.

\section{References}

[1] K.A.Pupkov, "Nestatsionarnye sistemy avtomaticheskogo upravleniya: analiz, sintez i optimizatsiya" ("Time-varying systems of automated control: Analysis, synthesis and optimization", Pupkov K.A. (Ed.)), Moscow, Izd-vo MGTU im.N.E. Baumana, 2007, pp. 100-117.

[2] N.P.Demenkov, G.N.Basilev, "Management of technical systems", Moscow: Izd-vo MGTU im.N.E. Baumana, 2013, pp. 116-128.

[3] L.B. Alekseeva, "Determination of transient behavion characteristics in drive control of machines", Gornyy informatsionno-analiticheskiy byulleten, vol. 4, pp. 18-25, 2016.
[4] A.D. Myshkis, "Elementy teorii matematicheskikh modeley" (Details of theory of mathematical models), Moscow: Komkniga, 2007, pp. 35-47.

[5] Uvarov V.P.Il'ichev V.A. "The effect of changes in process parameters on the stability of optical fiber drawing", Glass and Ceramics, vol. 5, pp. 21-22, 2003.

[6] V.P. Uvarov, "Standardization of process parameters rod drawing using experimental models", Glass and Ceramics, vol 11, pp. 8-9, 2003.

[7] M. Shtelle, R. Bryukner, "Predel'nye parametry protsessa vytyagivaniya steklovolokna", Clastechnische Berichte, vol. 5, pp. 130-139, 1980.

[8] M.D Morton, "Protsess nepreryvnoy vytyazhki vyazkikh zhidkostey pri pryadenii volokna", Ann. Rev. Fluid Mech., vol. 12, pp. 365-387, 1980.

[9] L.B. Alekseeva, V.V. Maksarov, "Research of stability of an automated control system for process of extracting optical rod drawing", Izvestiya vuzov. Mashinostroenie, vol. 8, pp. 19-24, 2008.

[10] V.S.Bessmertnyj, V.P. Krokhin, "Quantitative criteria for evaluation of glass viscosity”, Glass and Ceramics, vol. 11, pp. 11-13, 2001. 